\title{
A Simplified Model for the Optimization of LC Photonic Elements
}

\author{
Li Tan, Jacob Y. Ho, Abhishek K. Srivastava, and Hoi-Sing Kwok, Fellow, IEEE
}

\begin{abstract}
Recently, liquid crystal (LC) polarization gratings (PGs), because of several advantages, have become extremely popular although the process for analysis and optimization of different optical characteristics is still very complicated. Here, we reveal a simplified model (SM) to evaluate and optimize the performance of LCPGs. Unlike the existing extended Jones matrix (EJM) method, the SM excludes the layer-by-layer analysis and considers an effective twist that reduces the complexity of the calculations. In addition, obtained results from the SM have been compared with the EJM model for variety of conditions and found in good agreement. Furthermore, the proposed SM can also be used for variety of LC photonic elements viz., polarization convertors, polarization rotators, and so on.
\end{abstract}

Index Terms-Liquid crystal devices, diffraction, gratings.

\section{INTRODUCTION}

$\mathbf{P}$ OLARIZATION gratings (PGs) are defined as spatially varying dichroic or birefringent elements whose anisotropy parameters vary across the device periodically. The polarization state of the transmitted electric field is thus modulated periodically [1]. Because of its well-known interesting optical properties [2]-[5], a variety of applications have been proposed, including displays [6], polarimeters for measuring stokes parameters [7], [8] or Muller matrix [9], beam steering [10], [11], polarization conversion element [12] etc.

Reported fabrication methods of PGs include space-variant subwavelength-carrier surface-relief elements using electron beam lithography [13]. However, it is very difficult to achieve the grating periods $<100 \mu \mathrm{m}$ by using the fabrication method described in Ref. [13]. On the other hand, a method to obtain continuous in-plane axis variation of the birefringent medium through polarization holography between two coherent laser waves has been proposed [14]. The recording materials can be gelatin film containing photo-birefringent azobenzene [15], or LC photoalignment layers, such as polarization sensitive azodye-doped polyimide films [16], linear photo-polymerizable polymers [17] and azo-dye molecules [18], [19]. The spatial

Manuscript received October 29, 2013; revised February 16, 2014; accepted March 31, 2014. Date of publication April 10, 2014; date of current version April 29, 2014. This work was supported in part by the Hong Kong Government Research Grants Council under Grant 614410 and in part by the Partner State Key Laboratory on Advanced Displays and Optoelectronics Technologies, Hong Kong University of Science and Technology, Hong Kong.

The authors are with the Partner State Key Laboratory on Advanced Displays and Optoelectronics Technologies, Department of Electronic and Computer Engineering, Hong Kong University of Science and Technology, Hong Kong (e-mail: eetanli@ust.hk; eejho@ust.hk; abhishek_srivastava_lu@yahoo.co.in; eekwok@ust.hk).

Color versions of one or more of the figures in this letter are available online at http://ieeexplore.ieee.org.

Digital Object Identifier 10.1109/LPT.2014.2315492 profile recorded on the alignment materials was successfully transferred to a nematic LC bulk or LC polymer with proper thickness and grating pitch [18].

LCPGs have the advantages of good stability, easy fabrication, voltage controllable diffraction, flexibility of inducing different LC deformations [14], [17], [20]. The deformations in the bulk LCs and the complex PG configurations, such as the multilayer structure, provide additional flexibility to control different aspects of the fabricated PGs. This improves the design variety of PGs to meet the requirements of different applications, such as the broadband property, and/or wide viewing angle property desired in display systems, or beam steering systems etc.

However, the complex structures and deformations are hard to be characterized and optimized through analytical method. Several numerical methods based on complex analysis have been proposed. Finite-difference time-domain (FDTD) algorithm [21], [22] was used for the oblique incidence performance evaluations using the extended split-field update technique. However, it involves an abstruse processing and becomes substantially time consuming and complex for the comprehensive analysis of the different optical devices. In a recent effort, we developed a relatively faster and accurate simulation method using extended Jones matrix (EJM) combined with vectorial Fourier transformations [23]. It has the advantage of extension to system level performance simulation. However, the EJM method involves layer-by-layer analysis that demands to divide a device into a stack of $\mathrm{N}$-layers $(\mathrm{N}$ is typically around $50 \sim 100)$ during the simulation, which is relatively easier but still rather complicated for the complex and multiple component systems. Therefore, a simplified method with simple logical concepts is desired for optimizing complex structures of LC photonic elements including PG's.

In this article, we propose a simplified model (SM) to simulate the optical characteristics of the periodic twisted anisotropic LC photonic elements, particularly for the PG, at both ON and OFF axis incidence by considering the resultant twist that does not involve layer-by layer analysis of the LC. Using this approach, the characterization of LCPGs at oblique incidence is simplified to the level of normal incidence calculations. The transmitted field of passing through a PG at oblique incidence can be directly characterized by regular Jones matrix instead of extended Jones matrix. Thus, exclusion of the $\mathrm{N}$-sub layer consideration of the $\mathrm{LC}$ photonic element provides an opportunity to make the problem much simpler, effective and user friendly for the complex and multicomponent LC 


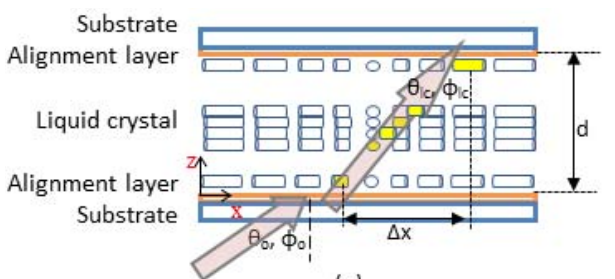

(a)

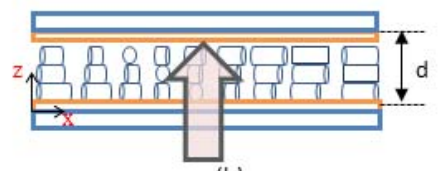

(b)

Fig. 1. Schematic side view of a primary type of LCPG at oblique incidence (a); and its approximate normal incidence model (b).

systems [24], [25]. Applying the SM together with parameter space diagram, we have efficiently optimized a complex type wide viewing angle (WVA) PG.

\section{Simplified Model OF LCPGS AT OBLIQUE INCIDENCE}

To clarify the proposed model and its effectiveness we have considered the structure of a LCPG cell. The schematic side view of a primary type of LCPG is shown in Fig. 1(a). It contains LC directors distributed periodically along the $\mathrm{x}$-axis. Light is incident on the grating obliquely from the air at an angle $\left(\theta_{o}, \Phi_{o}\right)$, and refracted into the LC layer obeying Snell's law.

There are two major effects that play crucial role for the diffraction. First, is the effective birefringence deviation $\Delta n_{e f f}=n_{e}(\theta)-n_{o}$, where $n_{e}(\theta)$ in terms of the angle between the light wave and the director c-axis is defined as follows:

$$
\frac{1}{n_{e}^{2}(\theta)}=\frac{\cos ^{2} \theta}{n_{e}^{2}}+\frac{\sin ^{2} \theta}{n_{o}^{2}}
$$

For the LC with homogenous director distribution within the $\mathrm{x}-\mathrm{y}$ plane, the birefringence deviation at off-axis is not obvious.

The second and more severe effect comes from director orientation, which is caused specifically by LC grating structures. Because of the director variation within the $x-y$ plane, the director orientation along the off-axis light path is different from the on-axis case. As shown in Fig. 1. (a), the director changes orientation as the light wave traverses the cell obliquely. The spatially varying director distribution leads to an additional equivalent twist $\Delta \Phi_{\text {twist }}$ along the light propagation direction. The value of $\Delta \Phi_{\text {twist }}$ depends on the grating pitch $\Lambda$, the grating thickness $d$, the light wave angle within the LC layer $\left(\theta_{l c}, \Phi_{l c}\right)$, as follows:

$$
\begin{aligned}
\Delta \Phi_{\text {twist }} & = \pm \frac{\pi \Delta x}{\Lambda} \\
\Delta x & =d \tan \theta_{l c} \cos \Phi_{l c}
\end{aligned}
$$

The sign " \pm " of $\Delta \Phi_{\text {twist }}$ depends on whether it is $+\pi x / \Lambda$ or $-\pi x / \Lambda$ of the grating direction distribution within the $\mathrm{x}-\mathrm{y}$ plane.
Because of the above mentioned effects, the original grating with configuration parameters $\left(\Delta n, d, \Phi_{\text {twist }}, \Lambda\right)$ at oblique incidence is transformed to an equivalent configuration with parameters $\left(\Delta n^{\prime}, d^{\prime}, \Phi_{\text {twist }}^{\prime}, \Lambda\right)$ at normal incidence. For a grating with period $\Lambda$ at the same scale of grating thickness $d$, the effect of $\Delta \Phi_{\text {twist }}$ dominates the off-axis deviation, the configuration can be further simplified to an SM model with parameters $\left(\Delta n, d, \Phi_{\text {twist }}^{\prime}, \Lambda\right)$, where $\Phi_{\text {twist }}^{\prime}=\Phi_{\text {twist }}+\Delta \Phi_{\text {twist }}$. An illustration of the SM model is shown in Fig. 1. (b).

Now, the problem of LCPG at oblique incidence is simplified to that of a normal incidence case through this SM model. Wave incident from a specific position and propagating through the whole grating could be described directly by $2 \times 2$ Jones matrix $M_{L C}$. Moreover, if the input axis is along X-axis, the transformation matrix $M_{L C 0}$ is given as below [26]:

$$
M_{L C 0}=\left[\begin{array}{cc}
a-j b & -c-j d \\
c-j d & a+j b
\end{array}\right]
$$

Where

$$
\begin{aligned}
& a=\cos \chi \cos \Phi_{t w i s t}^{\prime}+\frac{\Phi_{\text {twist }}^{\prime}}{\chi} \sin \chi \sin \Phi_{t w i s t}^{\prime} \\
& b=\frac{\delta}{\chi} \sin \chi \cos \Phi_{\text {twist }}^{\prime} \\
& c=\cos \chi \sin \Phi_{t w i s t}^{\prime}-\frac{\Phi_{t w i s t}^{\prime}}{\chi} \sin \chi \cos \Phi_{t w i s t}^{\prime} \\
& d=\frac{\delta}{\chi} \sin \chi \sin \Phi_{t w i s t}^{\prime}
\end{aligned}
$$

$\Phi_{\text {twist }}^{\prime}$ and $\delta(=\pi \Delta n d / \lambda$,$) are the equivalent twist angle$ and the retardation value of the LC grating along $\mathrm{z}$-axis respectively. Whereas $\chi^{2}=\Phi_{\text {twist }}^{\prime}+\delta^{2}$.

For light wave input at a position with input LC director at an angle $\Phi=\Phi(x, y)$ :

$$
M_{L C}=M_{L C}(x, y)=R(\Phi(x, y)) M_{L C 0} R(-\Phi(x, y))
$$

where $R(\Phi)$ is the rotation matrix.

After obtaining the space-variant near field distribution, the far field property is derived through calculating its vectorial Fourier coefficient. So the whole calculation is simplified to superimpose the space-variant transmitted field, under Fraunhofer approximation.

\section{Calculated Results}

To illustrate the validity of the proposed SM method, the primary type of one-dimensional LCPG, with the c-axis of LC direction periodically changing in-plane along the $\mathrm{x}$-axis, has been simulated and thereafter the obtained results have been compared with the existing, complex, EJM method and experiments.

Assuming that the in-plane azimuthal angle $\Phi$ equals to $\Phi(x)=\pi x / \Lambda$, the retardation equals half wave of blue light $(\lambda=452 \mathrm{~nm})$. We apply a LC material with $\Delta \mathrm{n} \approx 0.2$ in our simulation. Since the drop of $\pm 1^{s t}$ order diffraction efficiency along grating vector direction is dominant, we plot the oblique incidence performance along this direction only. The calculated results from SM (solid legends) are compared with the EJM method (solid lines), Fig. 2. (a) and (b) for different pitches and found in good agreement with each 

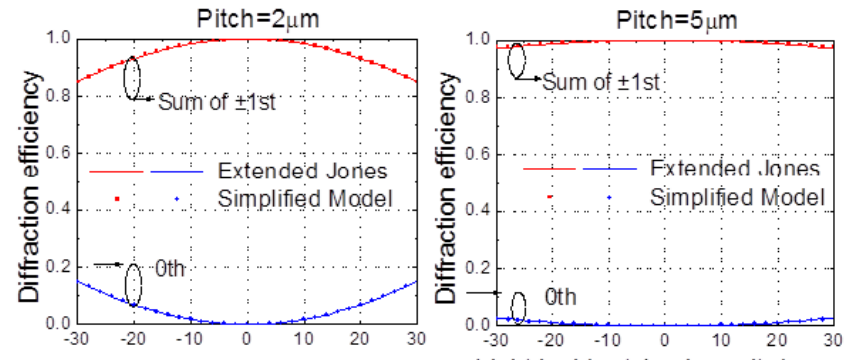

Light incident Angle, unit:degree Light incident Angle, unit:degree

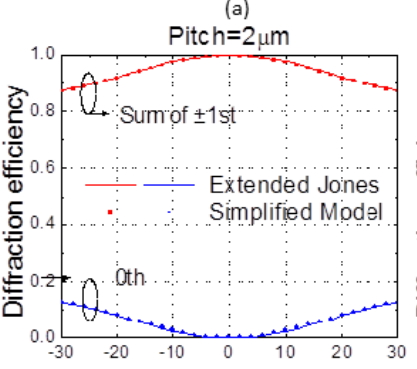

Light incident Angle, unit:degree

(c)

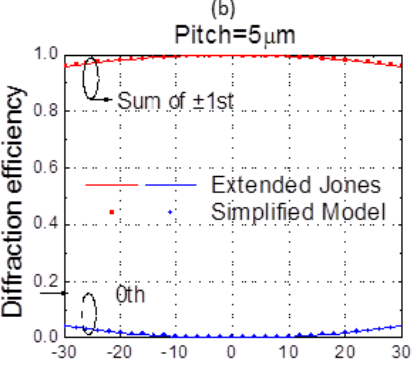

Light incident Angle, unit:degree

(d)

Fig. 2. Simulated viewing angle performance of primary types of LCPG. (a) $\Lambda=2.0 \mu \mathrm{m}$; (b) $\Lambda=5.0 \mu \mathrm{m}$ and complex types of LCPG with double layer opposite twist structure; (c) $\Lambda=2.0 \mu \mathrm{m}$; (d) $\Lambda=5.0 \mu \mathrm{m}$.

other. The computational time is is around 2.7 seconds and 153.7 seconds using SM and EJM method respectively coded using Matlab. Surface reflection is not considered for both calculations. Thus, the SM works well for the primary type of one-dimensional LCPGs.

Another more complex PG that involves double layer structure with opposite twist has also been simulated and thereafter compared with the results obtained from EJM in Fig. 2. (c) and (d). The computational time is around 4.6 seconds and 348.2 seconds using SM and EJM method respectively. This double layer structure was proposed previously to achieve achromatic diffraction [17]. Assuming that the twist angle of each layer equals 70 degree but with opposite twist sense, the retardation of each layer equals to $250 \mathrm{~nm}$ at $\lambda=550 \mathrm{~nm}$.

As illustrated from Fig. 2, results obtained from SM match well with the results obtained from EJM however the SM reduces the complexity of the calculation and the speedup the optimization (i.e. at least 100 times faster than EJM) that is critically important for the optimization of the multiple component systems [23].

\section{Parameter Space Optimization for PGs by SM}

The parameter space approach [26] is very useful in identifying the operating points and operating modes of various LC modes. Parameter space diagrams, usually showing the solution space of twist angle $(\Phi)$ and retardation $(\Delta n d)$, have many common display modes succinctly displayed on one graph. It provides a good illustration and prediction for optimizing display modes. With the ultra-fast algorithm derived in section 2, we can also analyze and optimize the PG configurations using the parameter space method.

For a certain PG, there are two main characteristics. First is the dispersion property, which means the diffraction property

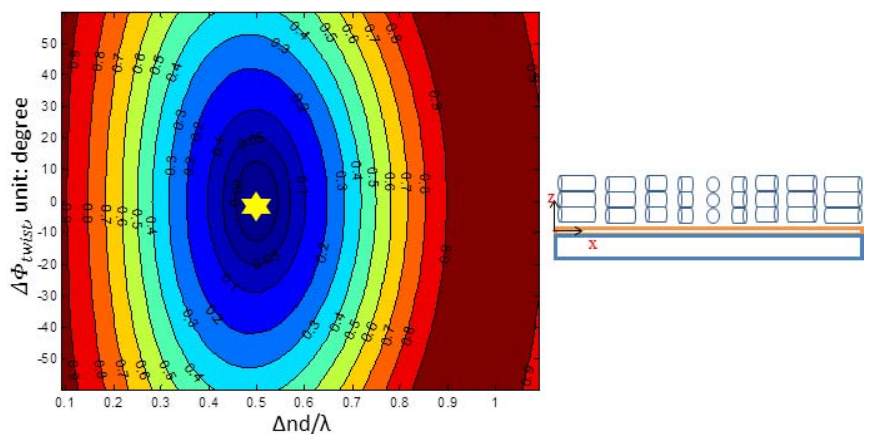

Fig. 3. Contour map of the $0^{\text {th }}$ order diffraction of a primary type PG, as a function of $\frac{\mathrm{d} \Delta \boldsymbol{n}}{\lambda}$ and $\Delta \Phi_{\mathrm{twist}}, \Lambda=2 \mu \mathrm{m}$.

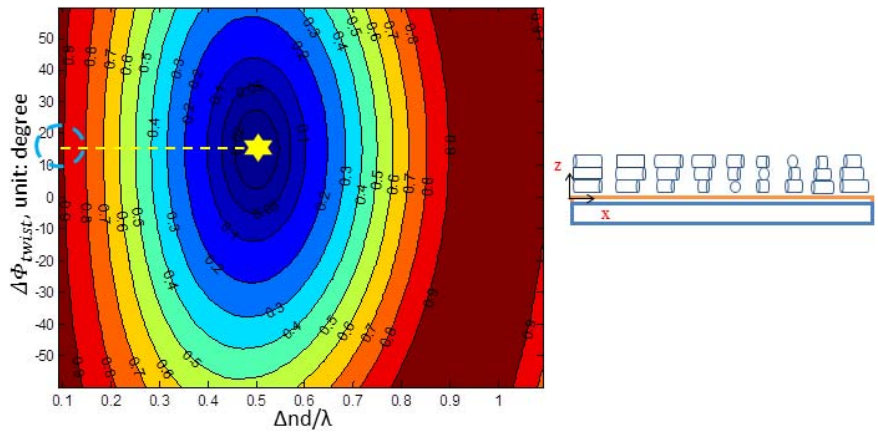

Fig. 4. Contour map of the $0^{\text {th }}$ order diffraction of a single layer PG with twist deformation, as a function of $\frac{\mathrm{d} \Delta \boldsymbol{n}}{\lambda}$ and $\Delta \Phi_{\mathrm{twist}}, \Lambda=2 \mu \mathrm{m}$.

at various wavelengths. It can be represented by the retardation value, $d \Delta n$ or $\frac{d \Delta n}{\lambda}$, since a change in $\lambda$ is the same as a change in $\mathrm{d} \Delta n$. The other is the viewing angle property, which means the diffraction property at different angle of incidence. According to $S M$, it is related to a deviation of twist, $\Delta \Phi_{t w i s t}$, superimposed to the PG twist deformation $\left(\Phi_{\text {twist }}\right)$.

For a primary type PG, the contour map of the $0^{\text {th }}$ order diffraction, as a function of $\frac{d \Delta n}{\lambda}$ and $\Delta \Phi_{t w i s t}$, is given in Fig. 3. The configuration optimized to achieve minimum $0^{\text {th }}$ order diffraction efficiency is located at the point of the yellow star. More importantly, the dispersion and viewing angle properties can be visualized easily through this map. The twist deformation involved in a single layer PG shifts the minimum $0^{\text {th }}$ order diffraction efficiency to an off axis angle of incidence as shown in Fig. 4.

We generated the parameter space diagrams as a function of $\frac{\Delta n d}{\lambda}$ and $\Delta \Phi$ of a series of PGs with different configurations.

Fig. 5 shows the case of a complex PG with double layer twist configuration when $\Phi_{1}=-\Phi_{2}=60^{\circ}$. We found that when $\frac{\Delta n d}{\lambda}=0.61$, it will not make the obviously $0^{\text {th }}$ order leakage increase over a large range of $\Delta \Phi$, as illustrated along the vertical yellow line in Fig. 5. It means that the grating maintains high diffraction efficiency into \pm 1 st order over a large range of angle of incidence, if ignoring the Fresnel reflection at interface.

We compared the $\sum \pm 1$ st diffraction efficiency as a function of angle of incidence of a primary type PG and a WVA PG as shown in Fig. 6 where the $\lambda=550 \mathrm{~nm}$ and $\Lambda=2 \mu \mathrm{m}$. For WVA PG, the $\Delta n d$ of each layer is set to be $336 \mathrm{~nm}$ $(=0.61 \times \lambda)$, while the $\Delta n d$ of primary type $\mathrm{PG}$ equals $275 \mathrm{~nm}$. 


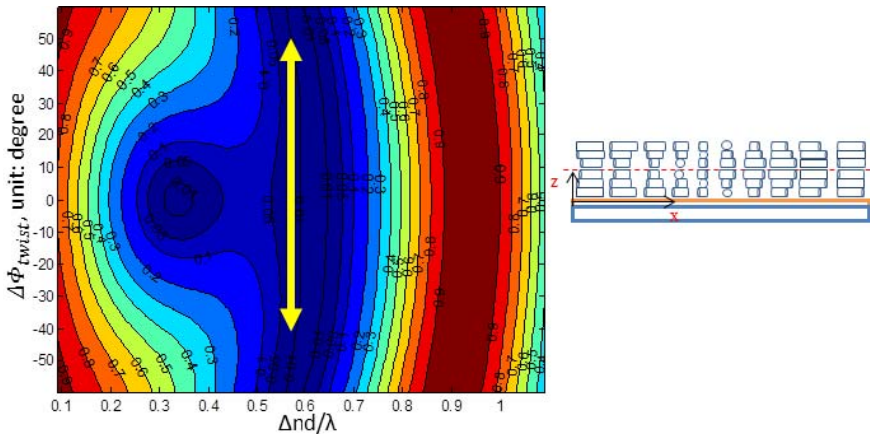

Fig. 5. Contour map of the 0th order diffraction of a WVA PG, $\Lambda=2 \mu \mathrm{m}$.

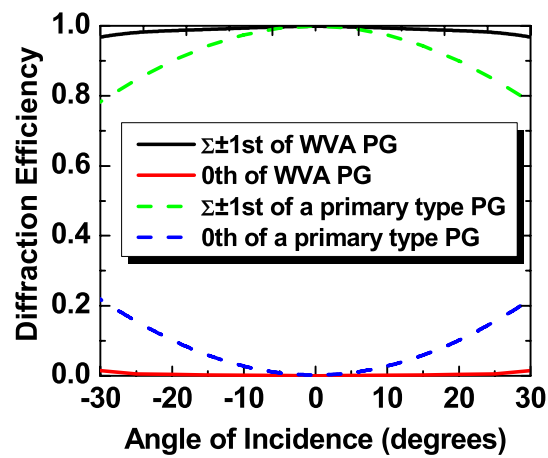

Fig. 6. Calculated diffraction efficiency at oblique incidence of a WVA PG and a primary type of PG at the pitch of $2 \mu \mathrm{m}, \lambda=550 \mathrm{~nm}$.

It is clear from the figure that at an incident angle of $20^{\circ}$, the WVA PG greatly suppressed the $0^{\text {th }}$ order leakage from $10 \%$ down to less than $0.5 \%$. The WVA PG is suitable for applications in monochromatic systems with light from a wide range of incident angle, such as a beam steering system. Results obtained from the SM match well with the previously reported results with EJM [23].

\section{CONCLUSIONS}

We have developed a simplified model to characterize the optical properties of LC photonic elements involving anisotropic director distribution of LC system. The SM is based on the consideration of the resultant twist that does not involve layer-by layer analysis of the LC and therefore make the optimization dramatically simpler and increases the simulation speed, which is critically important for the multi component devices. Moreover we revealed that the SM maintains its accuracy for both on and off axis situations and results match well with the widely accepted EJM model. Furthermore, a WVA PG was designed using the simplified model and parameter space method. Complex types of PGs provide additional adjustable parameters, which potentially improve the PGs' performance and greatly broaden their applications. Therefore, the proposed SM model provides effectives, user friendly and relatively faster approach for better optimizing complex types of LC photonic elements and therefore the SM could be implemented to optimize a range of more attractive properties and applications for the multicomponent LC photonic systems.

\section{REFERENCES}

[1] G. Cincotti, "Polarization gratings: Design and applications," IEEE J. Quantum Electron., vol. 39, no. 12, pp. 1645-1652, Dec. 2003.

[2] S. D. Kakichashvili, "Method for phase polarization recording of holograms," Soviet J. Quantum Electron., vol. 4, no. 6, pp. 795-798, 1974.

[3] S. D. Kakichashvili, "Polarization holography," Soviet Phys. Uspekhi, vol. 21, no. 1, pp. 998-1000, 1978.

[4] L. Nikolova and T. Todorov, "Diffraction efficiency and selectivity of polarization holographic recording," Opt. Acta, Int. J. Opt., vol. 31, no. 5, pp. $579-588,1984$.

[5] J. Tervo and J. Turunen, "Paraxial-domain diffractive elements with $100 \%$ efficiency based on polarization gratings," Opt. Lett., vol. 25, no. 11, pp. 785-786, 2000.

[6] R. K. Komanduri, C. Oh, and M. J. Escuti, "Polarization independent projection systems using thin film polymer polarization gratings and standard liquid crystal microdisplays," in SID Symp. Dig. Tech. Papers, vol. 40. 2009, pp. 487-490.

[7] F. Gori, "Measuring Stokes parameters by means of a polarization grating," Opt. Lett., vol. 24, no. 9, pp. 584-586, 1999.

[8] C. Provenzano, G. Cipparrone, and A. Mazzulla, "Photopolarimeter based on two gratings recorded in thin organic films," Appl. Opt., vol. 45, no. 17 , pp. 3929-3934, 2006.

[9] M. Kudenov, M. Escuti, N. Hagen, E. Dereniak, and K. Oka, "Snapshot imaging Mueller matrix polarimeter using polarization gratings," Opt. Lett., vol. 37, no. 8, pp. 1367-1369, 2012.

[10] J. Kim, C. Oh, S. Serati, and M. J. Escuti, "Wide-angle, nonmechanical beam steering with high throughput utilizing polarization gratings," Appl. Opt., vol. 50, no. 17, pp. 2636-2639, 2011.

[11] C. Oh, J. Kim, J. Muth, S. Serati, and M. J. Escuti, "High-throughput continuous beam steering using rotating polarization gratings," IEEE Photon. Technol. Lett., vol. 22, no. 4, pp. 200-202, Feb. 15, 2010.

[12] J. Kim, R. Komanduri, K. Lawler, D. Kekas, and M. Escuti, "Efficient and monolithic polarization conversion system based on a polarization grating," Appl. Opt., vol. 51, no. 20, pp. 4852-4857, 2012.

[13] I. Vartiainen, J. Tervo, J. Turunen, and M. Kuittinen, "Surface-relief polarization gratings for visible light," Opt. Express, vol. 18, no. 22, pp. 22850-22858, 2010.

[14] G. P. Crawford, J. N. Eakin, M. D. Radcliffe, A. Callan-Jones, and R. A. Pelcovits, "Liquid-crystal diffraction gratings using polarization holography alignment techniques," J. Appl. Phys., vol. 98, no. 12, p. $123102,2005$.

[15] T. Todorov, L. Nikolova, G. Stoilov, and B. Hristov, "Spectral Stokesmeter. 1. Implementation of the device," Appl. Opt., vol. 46, no. 27, pp. 6662-6668, 2007.

[16] C. Provenzano, P. Pagliusi, and G. Cipparrone, "Highly efficient liquid crystal based diffraction grating induced by polarization holograms at the aligning surfaces," Appl. Phys. Lett., vol. 89, no. 12, p. 121105, 2006.

[17] C. Oh and M. J. Escuti, "Achromatic diffraction from polarization gratings with high efficiency," Opt. Lett., vol. 33, no. 20, pp. 2287-2289, 2008.

[18] V. Presnyakov, K. Asatryan, T. Galstian, and V. Chigrinov, "Optical polarization grating induced liquid crystal micro-structure using azo-dye command layer," Opt. Express, vol. 14, no. 22, pp. 10558-10564, 2006.

[19] S. R. Nersisyan et al., "Study of azo dye surface command photoalignment material for photonics applications," Appl. Opt., vol. 49, no. 10, pp. 1720-1727, 2010.

[20] M. Honma and T. Nose, "Twisted nematic liquid crystal polarization grating with the handedness conservation of a circularly polarized state," Opt. Express, vol. 20, no. 16, pp. 18449-18458, 2012.

[21] C. Oh and M. J. Escuti, "Time-domain analysis of periodic anisotropic media at oblique incidence: An efficient FDTD implementation," Opt. Express, vol. 14, no. 24, pp. 11870-11884, 2006.

[22] C. Oh and M. J. Escuti, "Numerical analysis of polarization gratings using the finite-difference time-domain method," Phys. Rev. A, vol. 76, p. 043815 , Oct. 2007 .

[23] L. Tan, J. Y. Ho, and H.-S. Kwok, "Extended Jones matrix method for oblique incidence study of polarization gratings," Appl. Phys. Lett., vol. 101, no. 5, p. 051107, 2012.

[24] F. Fan, T. Du, A. K. Srivastava, W. Lu, V. Chigrinov, and H. S. Kwok, "Axially symmetric polarization converter using patterned liquid crystal quarter wave plate," Opt. Express, vol. 20, no. 21, p. 23036, 2012.

[25] H. Ren, Y. H. Lin, and S. T. Wu, "Linear to axial or radial polarization conversion using a liquid crystal gel," Appl. Phys. Lett., vol. 89, no. 5, p. 051114, 2006.

[26] H. S. Kwok, "Parameter space representation of liquid crystal display operating modes," J. Appl. Phys., vol. 80, no. 7, pp. 3687-3693, 1996. 